\title{
Collision Detection and Safe Reaction with the DLR-III Lightweight Manipulator Arm
}

\author{
Alessandro De Luca \\ Dipartimento di Informatica e Sistemistica \\ Università di Roma "La Sapienza" \\ Via Eudossiana 18, 00184 Roma, Italy \\ deluca@dis.uniroma1.it
}

\author{
Alin Albu-Schäffer Sami Haddadin Gerd Hirzinger \\ DLR - German Aerospace Center \\ Institute of Robotics and Mechatronics \\ P.O. Box 1116, D-82230 Wessling, Germany \\ \{alin.albu-schaeffer, sami.haddadin, gerd.hirzinger\}@dlr.de
}

\begin{abstract}
A robot manipulator sharing its workspace with humans should be able to quickly detect collisions and safely react for limiting injuries due to physical contacts. In the absence of external sensing, relative motions between robot and human are not predictable and unexpected collisions may occur at any location along the robot arm. Based on physical quantities such as total energy and generalized momentum of the robot manipulator, we present an efficient collision detection method that uses only proprioceptive robot sensors and provides also directional information for a safe robot reaction after collision. The approach is first developed for rigid robot arms and then extended to the case of robots with elastic joints, proposing different reaction strategies. Experimental results on collisions with the DLR-III lightweight manipulator are reported.
\end{abstract}

\section{INTRODUCTION}

Safety issues are of primary concern when robot manipulators are supposed to operate in an unstructured environment, sharing the workspace with a human user and allowing a close physical cooperation [1], [2]. Accidental collisions that may harm humans should be avoided by anticipating dangerous situations, while the effects of actual collisions should be mitigated by having the robot react promptly so as to recover a safe operative condition.

Current research on physical human-robot interaction deals with different aspects involved in robot dependability: mechanical design, aimed at reducing manipulator inertia and weight; introduction of compliant components, for reducing the severity of impacts; additional use of external sensors, so as to allow a fast detection of human-robot proximity; motion planning and control strategies, for minimizing the risks associated to collisions. Each of these aspects is relevant in one or more of the elementary phases in which a physical human-robot interaction can be divided.

In the pre-impact phase, collision avoidance is the primary goal and requires (at least, local) knowledge of the current environment geometry and computationally intensive motion planning techniques. Along a similar but simpler line, robot manufacturers are providing hw/sw facilities for prescribing safe Cartesian areas that should not be accessed by the robot in any operative condition. Anticipating incipient collisions or recognizing them in real-time is typically based on the use of additional external sensors, such as sensitive skins [3], onboard vision [4], strain gages, force load cells, and so on.
When a collision occurs, the resulting contact forces during the impact phase may be alleviated by pursuing a lightweight robot design [5], by adding soft visco-elastic covering to the links [6], or by introducing compliance in the driving system so as to mechanically decouple the heavy motor inertia from the link inertia [7]-[9]. Light but stiff link materials can be combined with harmonic drives, introducing thus joint elasticity, as in the DLR-III robot manipulator. Also, variable stiffness actuation can be used, stiffening the joints during low-velocity transients and relaxing them at high-velocity regimes [10], in such a way that tracking performance during free motion is not compromised by the introduced compliance.

In the post-impact phase, the first task is to detect the collision occurrence, which may have happened at any location along the robot arm. The controller should then switch to an appropriate reaction strategy, the most simple one being to stop the robot. It is obviously more cost effective to be able to detect a collision without the need of additional sensors. A rather intuitive scheme is to compare the commanded torque (or, the current in an electrical drive) with the nominal modelbased command (i.e., the torque expected in the absence of collision) and looking for fast transients due to possible collision [6], [11], [12]. This approach has been refined by including adaptive compliance control [13], [14]. However, tuning of collision detection thresholds in these schemes is difficult because of the highly varying dynamic characteristics of the commanded torques. Moreover, their common drawback (even when robot dynamics is perfectly known) is that the inverse dynamics computation for torque comparison is based on acceleration estimates that introduce noise (due to numerical differentiation of velocity or position data) and/or an intrinsic delay in a digital implementation.

A detection scheme that works under similar conditions and avoids the above drawbacks has been recently proposed in [15]. Collisions are viewed as faulty behaviors of the robot actuating system, while the design of a detector takes advantage of the decoupling property of robot generalized momentum [16], [17]. Moreover, this detection scheme is particularly convenient when it is necessary to switch control strategy, being independent from the control method used to generate the commanded motor torques.

In this paper, we build upon the original idea of [15] 
and present a complete treatment of the post-impact phase, from collision detection and identification to robot reaction strategies. In particular, the directional information on contact forces provided by the identification scheme is used to safely drive the robot away from the human. Section II recalls the physical properties of the robot total energy and of its generalized momentum which are relevant for our problem. New detection schemes and an analysis of the detectability of collision forces are presented in Section III for rigid manipulators. The extension to the case of robots with elastic joints, with alternative sets of reaction strategies, is discussed in Section IV. Finally, Section V reports on the experimental results obtained with the DLR-III lightweight manipulator.

\section{PRELIMINARIES}

We first consider robot manipulators as open kinematic chains of rigid bodies, having $N$ (rotational) rigid joints. The generalized coordinates $\boldsymbol{q} \in \mathbb{R}^{N}$ can be associated to the position of the links. The dynamic model is

$$
\boldsymbol{M}(\boldsymbol{q}) \ddot{\boldsymbol{q}}+\boldsymbol{C}(\boldsymbol{q}, \dot{\boldsymbol{q}}) \dot{\boldsymbol{q}}+\boldsymbol{g}(\boldsymbol{q})=\boldsymbol{\tau}_{\mathrm{tot}},
$$

where $\boldsymbol{M}(\boldsymbol{q})$ is the symmetric, positive definite inertia matrix, the Coriolis and centrifugal terms are factorized using the matrix $\boldsymbol{C}(\boldsymbol{q}, \dot{\boldsymbol{q}})$ of Christoffel symbols, and $\boldsymbol{g}(\boldsymbol{q})$ is the gravity vector. In the right-hand side of (1), $\tau_{\text {tot }}$ contains all active generalized torques performing work on $\boldsymbol{q}$ and all dissipative torques. In the absence of friction and other external torques acting from the environment, $\tau_{\text {tot }}$ is just the motor torque $\boldsymbol{\tau}$. From the skew-symmetry of matrix $\dot{\boldsymbol{M}}(\boldsymbol{q})-2 \boldsymbol{C}(\boldsymbol{q}, \dot{\boldsymbol{q}})$ it follows that

$$
\dot{\boldsymbol{M}}(\boldsymbol{q})=\boldsymbol{C}(\boldsymbol{q}, \dot{\boldsymbol{q}})+\boldsymbol{C}^{T}(\boldsymbol{q}, \dot{\boldsymbol{q}}) .
$$

The total energy of the robot is the sum of its kinetic energy and potential energy due to gravity:

$$
E=T+U=\frac{1}{2} \dot{\boldsymbol{q}}^{T} \boldsymbol{M}(\boldsymbol{q}) \dot{\boldsymbol{q}}+U_{g}(\boldsymbol{q}),
$$

with $\boldsymbol{g}(\boldsymbol{q})=\left(\partial U_{g}(\boldsymbol{q}) / \partial \boldsymbol{q}\right)^{T}$. From (1) and (2), it is

$$
\dot{E}=\dot{\boldsymbol{q}}^{T} \boldsymbol{\tau}_{\text {tot }},
$$

which represents the energy balance in the system.

The generalized momentum of the robot is defined as

$$
\boldsymbol{p}=\boldsymbol{M}(\boldsymbol{q}) \dot{\boldsymbol{q}} .
$$

Using again (1) and (2), its time evolution is given by

$$
\dot{\boldsymbol{p}}=\boldsymbol{\tau}_{\text {tot }}+\boldsymbol{C}^{T}(\boldsymbol{q}, \dot{\boldsymbol{q}}) \dot{\boldsymbol{q}}-\boldsymbol{g}(\boldsymbol{q}) .
$$

The $i$-th component of $\dot{\boldsymbol{p}}$ can also be written as

$$
\dot{p}_{i}=\tau_{\text {tot }, i}-\frac{1}{2} \dot{\boldsymbol{q}}^{T} \frac{\partial \boldsymbol{M}(\boldsymbol{q})}{\partial q_{i}} \dot{\boldsymbol{q}}-g_{i}(\boldsymbol{q}),
$$

for $i=1, \ldots, N$. The dynamics of the generalized momentum is thus decoupled component-wise with respect to the torques acting on the right-hand side of eq. (1).

In the second part of the paper, we will extend our results to the case of robots with rigid links but elastic joints/transmissions. For the dynamic modeling of elastic joint robots, a doubling of generalized coordinates is needed in a Lagrangian formulation. Let $\boldsymbol{\theta} \in \mathbb{R}^{N}$ be the motor positions (as reflected through the gearboxes) and $\boldsymbol{q} \in \mathbb{R}^{N}$ be the link positions. Elasticity of the motion transmission elements is modeled by linear springs introduced at each joint. We consider also joint viscosity effects as in [18]. With the standard assumptions in [19], the dynamic model becomes

$$
\begin{gathered}
\boldsymbol{M}(\boldsymbol{q}) \ddot{\boldsymbol{q}}+\boldsymbol{C}(\boldsymbol{q}, \dot{\boldsymbol{q}}) \dot{\boldsymbol{q}}+\boldsymbol{g}(\boldsymbol{q})=\boldsymbol{\tau}_{\mathrm{tot}, J} \\
\boldsymbol{B} \ddot{\boldsymbol{\theta}}+\boldsymbol{D} \boldsymbol{K}^{-1} \dot{\boldsymbol{\tau}}_{J}+\boldsymbol{\tau}_{J}=\boldsymbol{\tau},
\end{gathered}
$$

where

$$
\tau_{J}=\boldsymbol{K}(\boldsymbol{\theta}-\boldsymbol{q})
$$

is the elastic force transmitted through the joints. The motor inertia matrix $\boldsymbol{B}$ and the joint stiffness matrix $\boldsymbol{K}$ are diagonal and positive definite, while $\boldsymbol{D} \geq \mathbf{0}$ is the diagonal joint viscosity matrix. In the right-hand side of the first equation in (7), $\boldsymbol{\tau}_{\text {tot }, J}$ contains the torques performing work on $\boldsymbol{q}$, i.e., those transmitted through the visco-elastic joints and the external torques acting from the environment. When the latter are absent, it is $\boldsymbol{\tau}_{\text {tot }, J}=\boldsymbol{\tau}_{J}+\boldsymbol{D} \boldsymbol{K}^{-1} \dot{\boldsymbol{\tau}}_{J}=\boldsymbol{K}(\boldsymbol{\theta}-\boldsymbol{q})+$ $\boldsymbol{D}(\dot{\boldsymbol{\theta}}-\dot{\boldsymbol{q}})$. We note that the analysis presented in this paper applies, with minor modifications, also to the more complete model of robots with elastic joints considered, e.g., in [20].

\section{DETECTION AND REACTION WITH RIGID ROBOTS}

During normal operation, the robot arm may collide with a standing or moving person/obstacle in its workspace. For simplicity, we assume that there is at most a single link involved in the collision. Let

$$
\boldsymbol{V}_{K}=\left[\begin{array}{c}
\boldsymbol{v}_{K} \\
\boldsymbol{\omega}_{K}
\end{array}\right]=\left[\begin{array}{c}
\boldsymbol{J}_{K, \operatorname{lin}}(\boldsymbol{q}) \\
\boldsymbol{J}_{K, \text { ang }}(\boldsymbol{q})
\end{array}\right] \dot{\boldsymbol{q}}=\boldsymbol{J}_{K}(\boldsymbol{q}) \dot{\boldsymbol{q}} \in \mathbb{R}^{6}
$$

be the linear velocity at the contact point and the angular velocity of the associated robot link. The quantity $\boldsymbol{V}_{K}$, and in particular the (geometric) Jacobian $\boldsymbol{J}_{K}(\boldsymbol{q})$, is unknown in advance. Accordingly, the Cartesian collision forces and moments are denoted by

$$
\boldsymbol{F}_{K}=\left[\begin{array}{c}
\boldsymbol{f}_{K} \\
\boldsymbol{m}_{K}
\end{array}\right] \in \mathbb{R}^{6}
$$

When a collision occurs, the robot dynamics (1) becomes

$$
\boldsymbol{M}(\boldsymbol{q}) \ddot{\boldsymbol{q}}+\boldsymbol{C}(\boldsymbol{q}, \dot{\boldsymbol{q}}) \dot{\boldsymbol{q}}+\boldsymbol{g}(\boldsymbol{q})=\boldsymbol{\tau}+\boldsymbol{\tau}_{K},
$$

where the joint torque $\tau_{K}$ associated to the Cartesian collision (generalized) force $\boldsymbol{F}_{K}$ is given by

$$
\boldsymbol{\tau}_{K}=\boldsymbol{J}_{K}^{T}(\boldsymbol{q}) \boldsymbol{F}_{K}
$$

\section{A. Collision detection}

Define the scalar quantity

$$
\sigma(t)=k_{D}\left[E(t)-\int_{0}^{t}\left(\dot{\boldsymbol{q}}^{T} \boldsymbol{\tau}+\sigma\right) d s-E(0)\right],
$$

with $\sigma(0)=0, k_{D}>0$, and where $E(t)$ is the total robot energy at time $t \geq 0$, as defined in (3). Note that $\sigma$ can be 
computed using the measured joint position $\boldsymbol{q}$ and velocity $\dot{\boldsymbol{q}}$ (possibly obtained through numerical differentiation) and the commanded motor torque $\tau$. The latter may be the result of any type of control action. No acceleration measurement is needed.

Using eqs. (4) and (9), the resulting dynamics of $\sigma$ is

$$
\dot{\sigma}=-k_{D} \sigma+k_{D} \dot{\boldsymbol{q}}^{T} \boldsymbol{\tau}_{K}
$$

i.e., that of a first-order stable linear filter driven by the work performed by the joint torques due to collision. During free motion, $\sigma=0$ up to measurement noise and unmodeled disturbances. In response to a generic collision, $\sigma$ raises exponentially with a time constant $1 / k_{D}$ and detection occurs as soon as $|\sigma|>\sigma_{\text {low }}$, a suitable threshold whose actual value depends on the noise characteristics in the system. Dynamic thresholding can be used for avoiding false detection due to spurious spikes in noisy signals, as shown in [21]. When contact is lost, $\sigma$ rapidly returns to zero. Because of these properties, we call $\sigma$ a collision detection signal.

Not all possible collision situations are detected by this scheme. With the robot at rest $(\dot{\boldsymbol{q}}=\mathbf{0})$, the instantaneous value of $\boldsymbol{\tau}_{K}$ does not affect $\sigma$, whereas this will happen only when the robot starts moving. As a consequence, with the robot at rest, true impulsive collision forces/torques cannot be detected by this scheme, unless they are mechanically filtered by the presence of a soft covering of the arm. On the other hand, when the robot is in motion, collision can be detected provided that the Cartesian collision force produces motion at the contact. In fact, using eq. (10),

$$
\dot{\boldsymbol{q}}^{T} \boldsymbol{\tau}_{K}=\dot{\boldsymbol{q}}^{T} \boldsymbol{J}_{K}^{T}(\boldsymbol{q}) \boldsymbol{F}_{K}=\boldsymbol{V}_{K}^{T} \boldsymbol{F}_{K}=0 \Longleftrightarrow \boldsymbol{V}_{K} \perp \boldsymbol{F}_{K}
$$

As a simple instance, a lateral (horizontal) force due to a human colliding against a $2 R$ planar arm in motion in the vertical plane will not be detected, being fully compensated by the reaction forces of the manipulator structure. When evaluated in terms of reactive motions that the robot may take in response to this collision, such behavior of the detection scheme is rather natural. In fact, no possible robot motion would be able to reduce the force loading in this case. Suppose now to add a vertical joint axis at the base (obtaining a $3 R$ elbow-type robot) and let the second and third links be in motion in the vertical plane as before (i.e., with $\dot{q}_{1}=0$ ). The same previous lateral force will be felt initially only at the first joint $\left(\tau_{K, 1} \neq 0\right)$, which is however at rest, so that $\dot{\boldsymbol{q}}^{T} \boldsymbol{\tau}_{K}=\mathbf{0}$ and thus $\dot{\sigma}(0)=0$. Provided that the joint position controller is soft enough, the first joint will start moving in response to the collision before the contact force has been removed and detection may then occur.

\section{B. Collision identification}

The previous scheme does not provide any directional information on the Cartesian collision force, nor is able to identify which robot link has collided. To this purpose, following [15], we define the $N$-dimensional quantity

$\boldsymbol{r}(t)=\boldsymbol{K}_{I}\left[\boldsymbol{p}(t)-\int_{0}^{t}\left(\boldsymbol{\tau}+\boldsymbol{C}^{T}(\boldsymbol{q}, \dot{\boldsymbol{q}}) \dot{\boldsymbol{q}}-\boldsymbol{g}(\boldsymbol{q})+\boldsymbol{r}\right) d s-\boldsymbol{p}(0)\right]$

with $\boldsymbol{r}(0)=\mathbf{0}$, a diagonal gain matrix $\boldsymbol{K}_{I}>\mathbf{0}$, and where $\boldsymbol{p}(t)$ is the robot generalized momentum at time $t \geq 0$, as defined in (5). Vector $r$ can be computed using the measured $(\boldsymbol{q}, \dot{\boldsymbol{q}})$ and the commanded motor torque $\boldsymbol{\tau}$. In particular, no inversion of the inertia matrix is needed.

From eqs. (6) and (9), the dynamics of $r$ is

$$
\dot{\boldsymbol{r}}=-\boldsymbol{K}_{I} \boldsymbol{r}+\boldsymbol{K}_{I} \boldsymbol{\tau}_{K},
$$

or, component-wise in the Laplace domain,

$$
\frac{r_{j}(s)}{\tau_{K, j}(s)}=\frac{K_{I, j}}{s+K_{I, j}}, \quad j=1, \ldots, N,
$$

with the $N$ decoupled transfer functions having unitary gains.

For (each component of) $\boldsymbol{r}$, all the appealing properties of the scalar detection signal $\sigma$ hold as well. In particular, collision will now be detected when $\|\boldsymbol{r}\|>r_{\text {low }}$ or, by working component-wise, when there exists at least an index $j$, with $j=1, \ldots, N$, for which $\left|r_{j}\right|>r_{\text {low }, j}$. In ideal conditions,

$$
\boldsymbol{K}_{I} \rightarrow \infty \Rightarrow \boldsymbol{r} \approx \boldsymbol{\tau}_{K},
$$

which means in practice that the gains should be taken as large as possible. Moreover, $\boldsymbol{r}$ is sensitive to collisions even at $\dot{\boldsymbol{q}}=\mathbf{0}$. When the contact occurs on the $i$-th link of the robot kinematic chain, we have

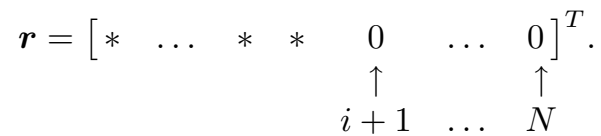

Assuming $\boldsymbol{r} \approx \boldsymbol{\tau}_{K}=\boldsymbol{J}_{K}^{T}(\boldsymbol{q}) \boldsymbol{F}_{K}$, this follows from the fact that, for a collision on link $i$, the last $N-i$ columns of the Jacobian $\boldsymbol{J}_{K}(\boldsymbol{q})$ are identically zero. In view of the structure (15), we call $\boldsymbol{r}$ a collision identification signal, or simply a residual bearing this term from the fault detection literature. The first $i$ components of vector $\boldsymbol{r}$ will be generically different from zero, at least for the time interval of contact, and will start decaying exponentially toward zero as soon as contact is lost. The residual $\boldsymbol{r}$ will be affected only by Cartesian collision forces $\boldsymbol{F}_{K}$ that perform virtual work on admissible robot motion, i.e., those forces that do not belong to the kernel of $\boldsymbol{J}_{K}^{T}(\boldsymbol{q})$. More in general, the sensitivity to $\boldsymbol{F}_{K}$ of each of the affected residuals (proximal to the robot base) will vary with the arm configuration (see also [15]). Thanks to the properties of the generalized momentum, this dynamic analysis can be carried out based only on the static transformation matrix $\boldsymbol{J}_{K}^{T}(\boldsymbol{q})$ from Cartesian forces to joint torques. In fact, the residual dynamics in eq. (14) is unaffected by robot velocity and acceleration.

\section{Reaction strategy}

In general, we suppose that the robot is always gravity compensated, i.e.,

$$
\boldsymbol{\tau}=\boldsymbol{\tau}^{\prime}+\boldsymbol{g}(\boldsymbol{q})
$$


with $\tau^{\prime}$ given by any motion control law. Enforcing such a zero-gravity condition is useful for guaranteeing a safe behavior [1], as robot motion will not be biased along the gravity direction. When motor torques are provided by a control law of the form (16), the computation of the detection signal $\sigma$ and of the residual $r$ may be simplified. In (11), $\tau$ can be replaced by $\tau^{\prime}$ and the total energy $E$ by the kinetic energy $T$, while in (13) one could just use $\boldsymbol{\tau}^{\prime}$ in place of $\boldsymbol{\tau}-\boldsymbol{g}(\boldsymbol{q})$. These modifications will not alter the resulting dynamics (12) (linear for $\sigma$ ) and (14) (linear and decoupled for $\boldsymbol{r}$ ).

During normal operation (pre-impact phase), it is convenient to apply a control law that provides accurate trajectory tracking in free motion while displaying passive properties (springdamper type) in response to unexpected collisions. A typical choice is a joint PD linear feedback law

$$
\boldsymbol{\tau}^{\prime}=\boldsymbol{\tau}_{d}^{\prime}+\boldsymbol{K}_{P}\left(\boldsymbol{q}_{d}-\boldsymbol{q}\right)-\boldsymbol{K}_{D} \dot{\boldsymbol{q}},
$$

with (diagonal) $\boldsymbol{K}_{P}>\mathbf{0}$ and $\boldsymbol{K}_{D}>\mathbf{0}$, and with the nominal feedforward term computed on the desired trajectory $\boldsymbol{q}_{d}(t)$ as

$$
\boldsymbol{\tau}_{d}^{\prime}=\boldsymbol{M}\left(\boldsymbol{q}_{d}\right) \ddot{\boldsymbol{q}}_{d}+\boldsymbol{C}\left(\boldsymbol{q}_{d}, \dot{\boldsymbol{q}}_{d}\right) \dot{\boldsymbol{q}}_{d}+\boldsymbol{K}_{D} \dot{\boldsymbol{q}}_{d} .
$$

The simplest reaction strategy to a collision would be to stop the robot by using its brakes [6]. However, this would not remove the arm from direct contact with a human, generating an unpleasant feeling of permanent danger or even squeezing the person in a narrow environment. Instead, the directional information embedded in the residual vector $\boldsymbol{r}$ can be used in the post-impact phase, by switching control to a more friendly behavior. In response to a collision, the residual $r$ rapidly increases and reaches peak values that depend on the severity of the impact. As soon as the detection threshold(s) is exceeded, the control law (17) is switched to the following simple reflex strategy:

$$
\boldsymbol{\tau}^{\prime}=\boldsymbol{K}_{R} \boldsymbol{r}, \quad \text { (diagonal) } \boldsymbol{K}_{R}>\mathbf{0} .
$$

The idea is to use the motor torques so as to (over)react to the external collision force along the same resulting direction, as seen at the level of joint torques. By combining eqs. (9), (16), and (18), and assuming the limit case of $\boldsymbol{r}=\boldsymbol{\tau}_{K}$, after collision detection the robot dynamics becomes

$$
\left(\boldsymbol{I}+\boldsymbol{K}_{R}\right)^{-1}(\boldsymbol{M}(\boldsymbol{q}) \ddot{\boldsymbol{q}}+\boldsymbol{C}(\boldsymbol{q}, \dot{\boldsymbol{q}}) \dot{\boldsymbol{q}})=\boldsymbol{\tau}_{K} .
$$

The robot inertial terms, as seen by a collision torque, are scaled by a factor larger than unity - a lighter pushable robot is obtained. In practice, because of the filtering introduced by eq. (14), this result will be obtained only partially. Equation (18) is clearly an active control scheme, as additional energy is feed into the system after the collision has been detected. The expected outcome, however, is that the robot bounces back in a direction (implicitly defined by eq. (18) and the robot arm dynamics) which is the most advantageous for escaping contacts, at least locally. When the contact is lost, and in the absence of further collisions, the residual will return to zero.

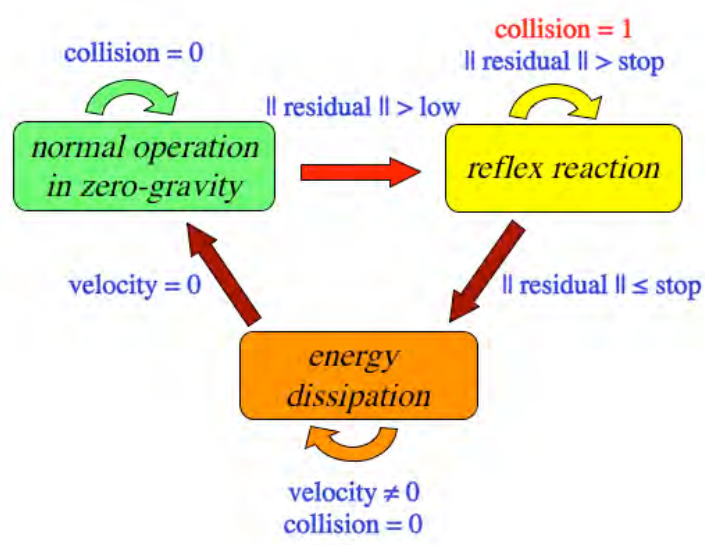

Fig. 1. Robot operation states

\section{Energy dissipation}

In the presence of very low friction, it may be necessary to limit the excursion of the robot reflex motion. In such cases, the control law (18) is only kept until $\|\boldsymbol{r}\| \leq r_{\text {stop }}$, when a phase of maximum dissipation of kinetic energy is executed in order to rapidly stop the robot in a safe configuration. Let the available motor torques at each joint be bounded by

$$
\left|\tau_{i}\right| \leq \tau_{\max , i}, \quad i=1, \ldots, N
$$

Part of this motor torque is spent for the gravity compensation in eq. (16). By defining the configuration-dependent bounds

$$
\begin{aligned}
\tau_{\mathrm{m}, i}^{\prime}(\boldsymbol{q}) & =-\left(\tau_{\max , i}+g_{i}(\boldsymbol{q})\right)<0 \\
\tau_{\mathrm{M}, i}^{\prime}(\boldsymbol{q}) & =\tau_{\max , i}-g_{i}(\boldsymbol{q})>0
\end{aligned}
$$

the remaining part of the available torques should satisfy

$$
\tau_{\mathrm{m}, i}^{\prime}(\boldsymbol{q}) \leq \tau_{i}^{\prime} \leq \tau_{\mathrm{M}, i}^{\prime}(\boldsymbol{q}), \quad i=1, \ldots, N
$$

Since the time evolution of the kinetic energy is $\dot{T}=\dot{\boldsymbol{q}}^{T} \boldsymbol{\tau}^{\prime}$, the following control law locally realizes the largest decrease of $T$ :

$$
\tau_{i}=\left\{\begin{array}{cl}
\tau_{\mathrm{m}, i}^{\prime}(\boldsymbol{q}) & \text { if } \dot{q}_{i} \geq \varepsilon_{i} \\
\tau_{\mathrm{m}, i}^{\prime}(\boldsymbol{q}) \dot{q}_{i} / \varepsilon_{i} & \text { if } \varepsilon_{i}>\dot{q}_{i} \geq 0 \\
\tau_{\mathrm{M}, i}^{\prime}(\boldsymbol{q}) \dot{q}_{i} / \varepsilon_{i} & \text { if }-\varepsilon_{i}<\dot{q}_{i} \leq 0 \\
\tau_{\mathrm{M}, i}^{\prime}(\boldsymbol{q}) & \text { if } \dot{q}_{i} \leq-\varepsilon_{i}
\end{array}\right\}+g_{i}(\boldsymbol{q}),
$$

with $i=1, \ldots, N$. For each velocity $\dot{q}_{i}$, the insertion of a small ultimate region of amplitude $2 \varepsilon_{i}>0$ allows a tradeoff between the almost minimum-time solution and a smooth reaching of the final condition $\dot{\boldsymbol{q}}=\mathbf{0}$.

The robot operation states and their transition conditions are shown in Fig 1. Note that as long as the collision flag is up, any further collision will keep the robot in the reflex reactive state. In other terms, the generator of the collision residual $r$ in eq. (13) is permanently active. 


\section{EXTENSION TO ROBOTS WITH JOINT ELASTICITY}

For a serial robot manipulator with significant joint/transmission elasticity, when a collision occurs the dynamic model (7) becomes

$$
\begin{aligned}
\boldsymbol{M}(\boldsymbol{q}) \ddot{\boldsymbol{q}}+\boldsymbol{C}(\boldsymbol{q}, \dot{\boldsymbol{q}}) \dot{\boldsymbol{q}}+\boldsymbol{g}(\boldsymbol{q}) & =\boldsymbol{\tau}_{J}+\boldsymbol{D} \boldsymbol{K}^{-1} \dot{\boldsymbol{\tau}}_{J}+\boldsymbol{\tau}_{K} \\
\boldsymbol{B} \ddot{\boldsymbol{\theta}}+\boldsymbol{D} \boldsymbol{K}^{-1} \dot{\boldsymbol{\tau}}_{J}+\boldsymbol{\tau}_{J} & =\boldsymbol{\tau}
\end{aligned}
$$

with $\boldsymbol{\tau}_{K}=\boldsymbol{J}_{K}^{T}(\boldsymbol{q}) \boldsymbol{F}_{K}$ as before.

The extension of the collision detection and identification schemes developed in Sect. III for the rigid case can be made in different ways. One possibility is to work with the complete energy of the robot, by including the kinetic energy of the motors and the potential energy associated to the joint elasticity in the definition of a detection signal $\sigma_{\mathrm{EJ}}$, or by considering also the generalized momentum associated to the motors in the definition of a residual $\boldsymbol{r}_{\mathrm{EJ}}$. Such schemes would in principle require the measure of the whole state $(\boldsymbol{\theta}, \dot{\boldsymbol{\theta}}, \boldsymbol{q}, \dot{\boldsymbol{q}})$ of the robot, together with the knowledge of the commanded torque $\tau$.

However, in view of the application of our methods to the DLR-III lightweight manipulator, we take advantage of the specific sensing devices available on board of this robot. In particular, every joint is equipped with a high-resolution incremental position sensor on the motor side and an integrated joint torque sensor, so that $\boldsymbol{\theta}$ and $\tau_{J}$ are directly available. From eq. (8), the link position is computed as $\boldsymbol{q}=\boldsymbol{\theta}-\boldsymbol{K}^{-1} \boldsymbol{\tau}_{J}$. Finally, $\dot{\boldsymbol{q}}$ and $\dot{\boldsymbol{\tau}}_{J}$ are obtained by numerical differentiation.

As a result, it is possible to consider only eq. (20) and handle the measured joint torque $\tau_{J}$ as the equivalent of the commanded torque $\boldsymbol{\tau}$ in the rigid robot model (9). More specifically, substituting $\boldsymbol{\tau}$ with $\boldsymbol{\tau}_{J}+\boldsymbol{D} \boldsymbol{K}^{-1} \dot{\boldsymbol{\tau}}_{J}$ in the expressions (11) and (13), we obtain similar collision detection schemes and identification properties. In particular, the residual $\boldsymbol{r}_{\mathrm{EJ}} \in \mathbb{R}^{N}$ for the visco-elastic joint case is defined as

$$
\begin{aligned}
\boldsymbol{r}_{\mathrm{EJ}}(t)=\boldsymbol{K}_{I} & {\left[\boldsymbol{p}(t)-\int_{0}^{t}\left(\boldsymbol{\tau}_{J}+\boldsymbol{D} \boldsymbol{K}^{-1} \dot{\boldsymbol{\tau}}_{J}\right.\right.} \\
& \left.\left.+\boldsymbol{C}^{T}(\boldsymbol{q}, \dot{\boldsymbol{q}}) \dot{\boldsymbol{q}}-\boldsymbol{g}(\boldsymbol{q})+\boldsymbol{r}_{\mathrm{EJ}}\right) d s-\boldsymbol{p}(0)\right],
\end{aligned}
$$

with $\boldsymbol{r}_{\mathrm{EJ}}(0)=\mathbf{0}$ and a (diagonal) gain matrix $\boldsymbol{K}_{I}>\mathbf{0}$. This leads again to the linear and decoupled residual dynamics

$$
\dot{\boldsymbol{r}}_{\mathrm{EJ}}=-\boldsymbol{K}_{I} \boldsymbol{r}_{\mathrm{EJ}}+\boldsymbol{K}_{I} \boldsymbol{\tau}_{K}
$$

\section{A. DLR-III controller}

We recall first the form of the robot control law used for general tasks with the DLR-III arm, in which reaction strategies to collisions have been inserted as additional control modalities. An interesting feature of this general controller, used already for Cartesian compliance control [18], is the possibility of shaping the motor inertia by a joint torque feedback of the form

$$
\begin{aligned}
\boldsymbol{\tau}= & \boldsymbol{B} \boldsymbol{B}_{\theta}^{-1} \boldsymbol{u}+\left(\boldsymbol{I}-\boldsymbol{B} \boldsymbol{B}_{\theta}^{-1}\right) \boldsymbol{\tau}_{J} \\
& +\left(\boldsymbol{D}-\boldsymbol{B} \boldsymbol{B}_{\theta}^{-1} \boldsymbol{D}_{s}\right) \boldsymbol{K}^{-1} \dot{\boldsymbol{\tau}}_{J}
\end{aligned}
$$

where $\boldsymbol{u} \in \mathbb{R}^{N}$ is the new torque command, $\boldsymbol{B}_{\theta}$ is the desired (still diagonal) motor inertia, with $\mathbf{0}<\boldsymbol{B}_{\theta}<\boldsymbol{B}$, and $\boldsymbol{D}_{s}>\boldsymbol{0}$ is the desired joint torque damping (diagonal) matrix. Substituting eq. (23) in (21) yields

$$
\boldsymbol{B}_{\theta} \ddot{\boldsymbol{\theta}}+\boldsymbol{D}_{s} \boldsymbol{K}^{-1} \dot{\boldsymbol{\tau}}_{J}+\boldsymbol{\tau}_{J}=\boldsymbol{u} .
$$

As a result, the inertia of the robot motors appears to be reduced - a convenient property for physical human-robot interaction. In fact, the motor inertia $\boldsymbol{B}$ is a significant part of the total inertia of the DLR-III manipulator: by reducing it to $\boldsymbol{B}_{\theta}$, the impact energy of the robot at a given velocity is scaled accordingly. Moreover, the effect of friction will be also reduced, allowing the human to move the robot by applying very low external forces.

A passivity-based controller, similar to eq. (17) of the rigid case, is obtained by choosing

$$
\boldsymbol{u}=\boldsymbol{\tau}_{J, d}+\boldsymbol{K}_{\theta}\left(\boldsymbol{\theta}_{d}-\boldsymbol{\theta}\right)-\boldsymbol{D}_{\theta} \dot{\boldsymbol{\theta}},
$$

with positive definite, diagonal matrices $\boldsymbol{K}_{\theta}$ and $\boldsymbol{D}_{\theta}$. The feedforward joint torque $\boldsymbol{\tau}_{J, d}$ and the motor reference $\boldsymbol{\theta}_{d}$ can be directly computed from the desired link trajectory $\boldsymbol{q}_{d}(t)$ (see, e.g., [22]). We note that the passivity properties are preserved provided that the following condition on the introduced (diagonal) damping matrices is satisfied [18]:

$$
\boldsymbol{D} \geq \frac{1}{4}\left(\boldsymbol{D}_{s}-\boldsymbol{D}\right)^{T} \boldsymbol{D}_{\theta}\left(\boldsymbol{D}_{s}-\boldsymbol{D}\right)
$$

Combining eqs. (23) and (24) yields the overall controller

$\boldsymbol{\tau}=\boldsymbol{K}_{P}\left(\boldsymbol{\theta}_{d}-\boldsymbol{\theta}\right)-\boldsymbol{K}_{D} \dot{\boldsymbol{\theta}}+\boldsymbol{K}_{P \tau}\left(\boldsymbol{\tau}_{J, d}-\boldsymbol{\tau}_{J}\right)-\boldsymbol{K}_{D \tau} \dot{\boldsymbol{\tau}}_{J}+\boldsymbol{\tau}_{J, d}$,

where the expressions of the final gain matrices can be easily obtained from the previous definitions. Equation (25) represents a full-state feedback with respect to suitable, possibly time-varying, reference values. Having an explicit joint torque feedback in addition to a position feedback loop, the above control law can be parametrized by a suitable choice of its gains so as to yield a position, a torque, or an impedance controller [23]. This fact will be used for implementing different strategies of robot reaction to collisions in a single framework.

Realizing a zero-gravity condition for an elastic joint robot, with a choice equivalent to eq. (16) of the rigid case, is not as immediate. Adding just $\boldsymbol{g}(\boldsymbol{q})$ in the expression of $\boldsymbol{\tau}$ is not enough to completely eliminate gravity effects from the picture. Rather, it can be shown that the more complex dynamic term $\boldsymbol{g}(\boldsymbol{q})+\boldsymbol{B} \boldsymbol{K}^{-1} \ddot{\boldsymbol{g}}(\boldsymbol{q})$ would be needed. However, it is often sufficient to compensate gravity in any static configuration. This can be more easily achieved by adopting the iterative scheme of [24] where, for each motor measurement $\boldsymbol{\theta}$, a gravity term $\overline{\boldsymbol{g}}(\boldsymbol{\theta})$ is computed such that

$$
\overline{\boldsymbol{g}}(\boldsymbol{\theta})=\boldsymbol{g}(\boldsymbol{q}), \quad \forall(\boldsymbol{\theta}, \boldsymbol{q}) \in \Omega:=\{(\boldsymbol{\theta}, \boldsymbol{q}) \mid \boldsymbol{K}(\boldsymbol{\theta}-\boldsymbol{q})=\boldsymbol{g}(\boldsymbol{q})\} .
$$

This approach fits well within the passivity framework of the controller. Another feasible scheme for on-line (partial) compensation of gravity has been proposed in [9]. 


\section{B. Reaction strategies}

The residual $\boldsymbol{r}_{E J}$ in eq. (22) is used for detecting a collision and identifying a safe direction for the reactive motion of the robot. In the presence of joint elasticity, however, a number of alternative strategies can be realized. As baseline behaviors in the performed collision experiments, we have taken the case of no reaction at all (Strategy 0) and of immediate stop of the trajectory generation with simultaneous high-gain position control (Strategy 1). With reference to the general control law (25), three reactive strategies have been considered.

\section{- Zero-gravity torque reaction (Strategy 2)}

$$
\boldsymbol{\tau}_{J, d}=\overline{\boldsymbol{g}}(\boldsymbol{\theta}), \quad \boldsymbol{K}_{P}=\mathbf{0} .
$$

This strategy leaves the robot floating in space in response to the collision force, while motion is damped at the motor side.

\section{- Reflex torque reaction (Strategy 3)}

$$
\boldsymbol{\tau}_{J, d}=\boldsymbol{K}_{R} \boldsymbol{r}_{\mathrm{EJ}}+\overline{\boldsymbol{g}}(\boldsymbol{\theta}), \quad \boldsymbol{K}_{P}=\mathbf{0} .
$$

This strategy is the closest to eq. (18) of the rigid case. Similar considerations can be made on the reduction of the apparent total inertia. In the limit case of $\boldsymbol{K}_{R}=\mathbf{0}$, this strategy collapses in the previous one.

\section{- Admittance control reaction (Strategy 4)}

$$
\boldsymbol{\tau}_{J, d}=\overline{\boldsymbol{g}}(\boldsymbol{\theta}), \quad \dot{\boldsymbol{\theta}}_{d}=\boldsymbol{K}_{R} \boldsymbol{r}_{\mathrm{EJ}} .
$$

In this case, the defined motor reference velocity $\dot{\boldsymbol{\theta}}_{d}$ is integrated so as to provide $\boldsymbol{\theta}_{d}$.

\section{EXPERIMENTS WITH THE DLR-III MANIPULATOR}

We have performed several tests on collision detection with the DLR-III lightweight manipulator and using the robot reaction strategies defined in Section IV-B.

Size and power of the DLR-III manipulator are fairly similar to that of a human arm, with an outreach of about $1 \mathrm{~m}$. The robot has 7 rotary joints, with spherical shoulder and wrist axes and a modular link/joint structure, a total weight of about $13.5 \mathrm{~kg}$, including brushless DC motors, electronics, and power converters (all integrated in the arm), and Harmonic Drives gear units with reduction ratios of 160 (100 for joint 5) and stiffnesses $K_{i}$ in the range $10 \div 15 \cdot 10^{3} \mathrm{Nm} / \mathrm{rad}$. The maximum output torques delivered by the motors range from $180 \mathrm{Nm}$ (first two joints) to $80 \mathrm{Nm}$ (joints 3 and 4), down to $30 \mathrm{Nm}$ (last three joints). The maximum joint velocities exceed $100^{\circ} / \mathrm{s}$, corresponding to a maximum attainable linear velocity at the end-effector larger than $4 \mathrm{~m} / \mathrm{s}$. The robot is equipped with electromagnetic emergency brakes. The highlevel control loop is implemented at $1 \mathrm{~ms}$ rate, with local joint control acting every $333 \mu \mathrm{s}$.

A preliminary evaluation of the HIC index [7] has been carried out using a dummy head instrumented with a threedimensional accelerometer [25]. We report here numerical results obtained on repeated collisions with a balloon (see Figs. 2-6) and qualitative results for collisions with humans (see Fig. 7).

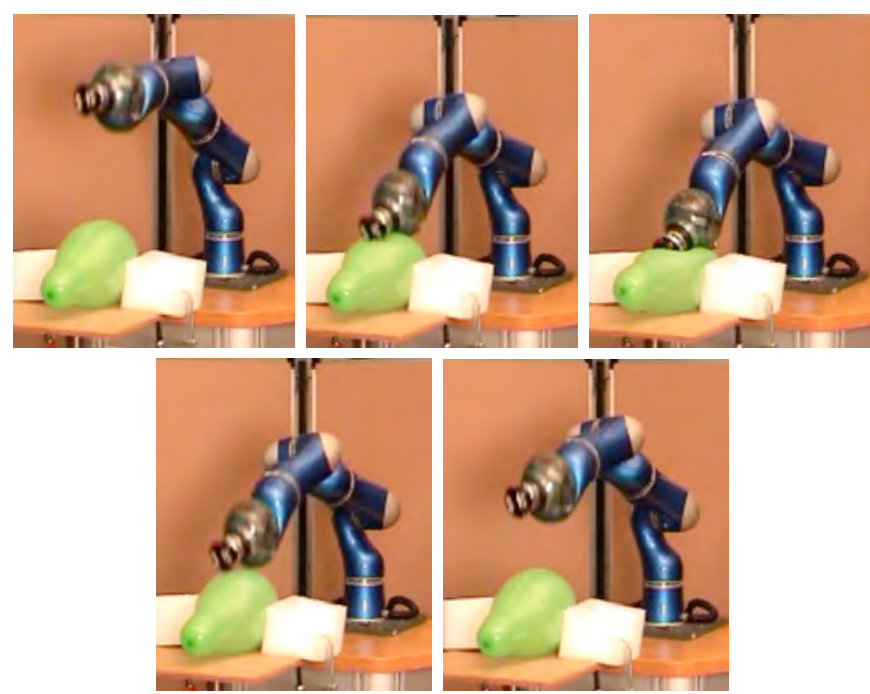

Fig. 2. Collision with a balloon (motion at $100 \%$ s) and robot reaction

The balloon experiment was chosen for its repeatable conditions needed to compare different reaction strategies. Tests were performed using a trapezoidal velocity profile, with cruise speed between $10 \%$ (slow speed impact) and $100^{\circ} / \mathrm{s}$ (fast speed impact), as reference trajectory $\boldsymbol{\theta}_{d}(t)$ for the motors. The stretched upward arm posture corresponds to $\boldsymbol{\theta}=\boldsymbol{q}=\mathbf{0}$ (gravity is zero in this configuration). Motion was planned between an initial and a final position specified by $\boldsymbol{\theta}_{\text {in }}=\left[\begin{array}{lllllll}60 & 31 & -78 & 23 & 158 & -15 & -15\end{array}\right]^{T}(\mathrm{deg})$ and $\boldsymbol{\theta}_{\mathrm{fin}}=\left[\begin{array}{lllllll}60 & 65 & -78 & 53 & 158 & -15 & -15\end{array}\right]^{T}$, respectively (motion is thus limited to joints 2 and 4 ). The robot hits the clamped balloon with its spherical wrist while coming from above (Fig. 2).

The detection gains were chosen as $\boldsymbol{K}_{I}=25 \cdot \boldsymbol{I}$, while the reflex reaction gains were selected as $\boldsymbol{K}_{R}=1.4 \cdot \boldsymbol{I}$ (for Strategy 3) or $\boldsymbol{K}_{R}=0.05 \cdot \boldsymbol{I}$ (for Strategy 4). The componentwise detection thresholds $r_{\text {low }, j}, j=1, \ldots, 7$, have been set at $10 \%$ of the maximum output torques available at each joint. This guarantees a reliable detection without false alarms and with just a one-step delay (1 ms). However, current control communication timings in the system set-up introduce an additional delay in the order of $4-6 \mathrm{~ms}$.

When no detection is activated (Strategy 0), the robot ends its motion while pressing the balloon, with an associated torque of $35 \mathrm{Nm}$ at joint 4 . Detection followed by stopping (Strategy 1) results in a residual balloon compression, which is slightly larger in the case of fast impact. For the other three reactive strategies, the backward robot motion leads in all cases to a reduction of the contact torque to zero.

For the impacts at slow speed (Figs. 3-4), the admittance control reaction is the fastest one, because a jump in the motor velocity is commanded (Strategy 4). Due to its active part, Strategy 3 reacts and reverts velocity slightly faster than Strategy 2. On the other hand, the reflex torque strategy drives back the robot for a longer movement, which would require the intervention the kinetic energy dissipation phase (not yet 


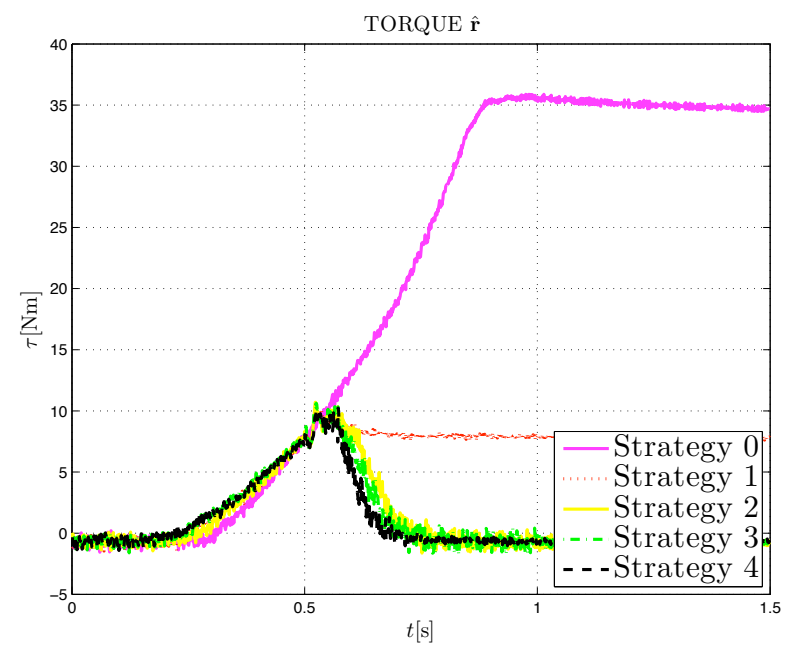

Fig. 3. Residual $r_{4}$ at joint 4 with different reaction strategies (impact at slow speed $=10^{\circ} / \mathrm{s}$ )

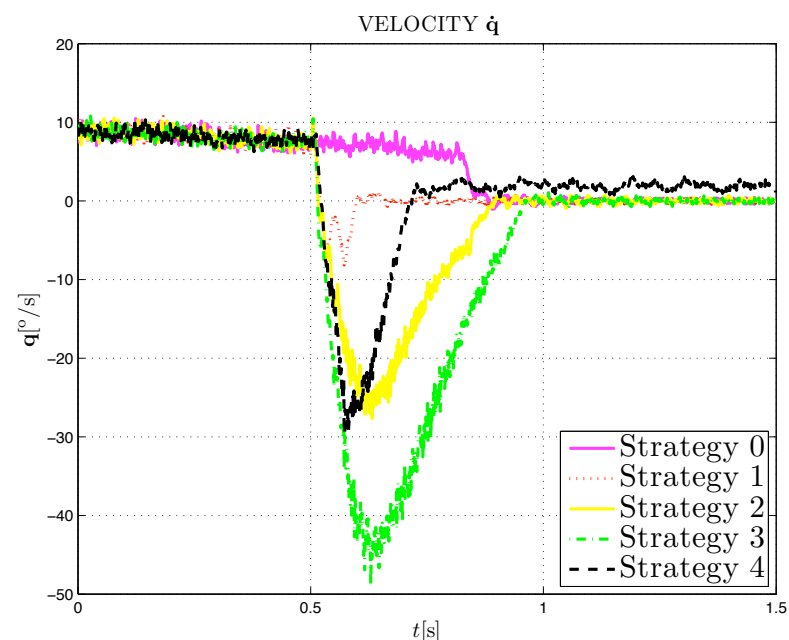

Fig. 4. Velocity $\dot{q}_{4}$ at joint 4 with different reaction strategies (impact at slow speed $=10^{\circ} / \mathrm{s}$ )

implemented).

For the impacts at fast speed (Figs. 5-6), a similar situation is observed. However, Strategies 1 and 4 are now the fastest in reversing motion, whereas the two reaction strategies in torque mode are not able to reduce the peak contact torque. Still, Strategy 3 is faster than 2 in driving the robot out of the collision area.

A similar behavior has been observed in the case of human-robot collisions. Robot motion was executed at 40 , 60 , and $90 \%$ of cruising joint velocity, starting from $\boldsymbol{\theta}_{\text {in }}=\left[\begin{array}{lllllll}90 & 45 & 0 & 90 & 0 & 0 & 0\end{array}\right]^{T}(\mathrm{deg})$ and heading to $\boldsymbol{\theta}_{\text {fin }}=\left[\begin{array}{lllllll}41.5 & 84.5 & 0 & -10 & 0 & 0 & 0\end{array}\right]^{T}$. Collisions occurred between the hand or the outstretched arm of different test persons and different locations between the 4-th and 6th link of the robot, with linear speeds at the contact up to approximately $1.5 \mathrm{~m} / \mathrm{s}$. The human arm is a relatively

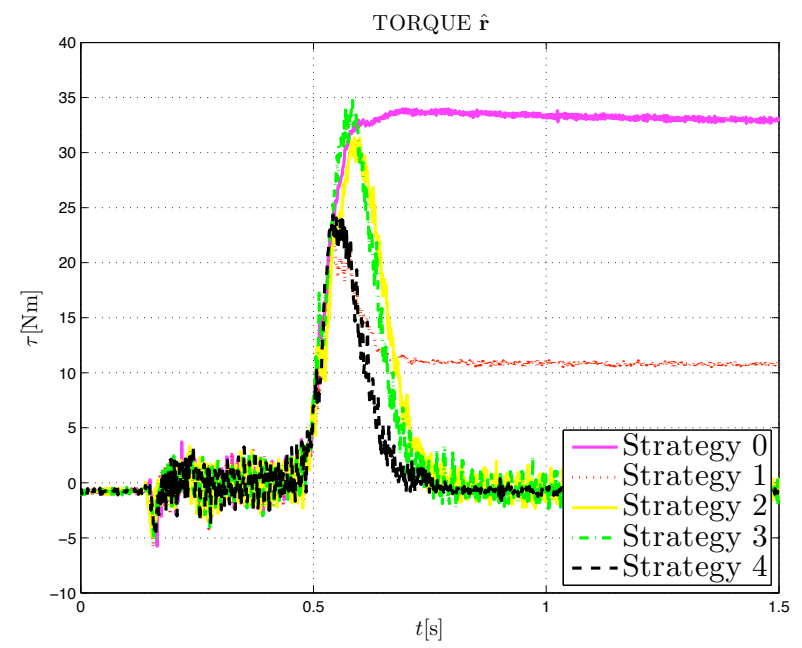

Fig. 5. Residual $r_{4}$ at joint 4 with different reaction strategies (impact at fast speed $=100^{\circ} / \mathrm{s}$ )

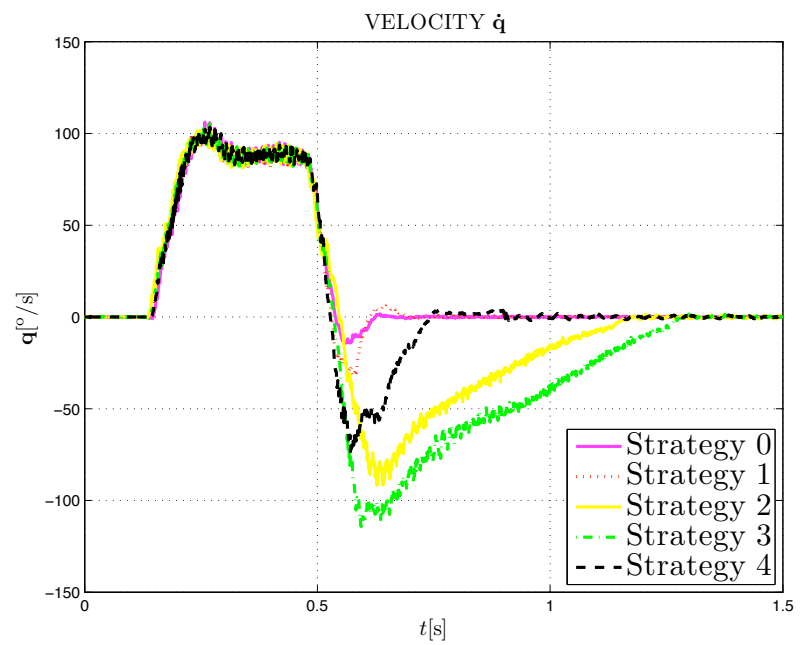

Fig. 6. Velocity $\dot{q}_{4}$ at joint 4 with different reaction strategies (impact at fast speed $=100^{\circ} / \mathrm{s}$ )

compliant structure which is free to move back in space in response to a collision. When the time interval of contact is relatively long, the detection and reaction capabilities of the robot are enhanced. After the first collision, the manipulator remains in the reactive mode so that the test person can start pushing it further away. The smoothest manual pushing of the robot arm was obtained when using Strategy 3. Videos of these experiments can be found on the web at http: //www.robotic.dlr.de/safe-robot.

Indeed, in order to evaluate accurately the impact forces in human-robot physical interaction, a suitable instrumented robot should be used during testing, e.g., including a force/torque sensor and accelerometers at a predefined collision point on the arm. However, beyond quantitative measures, the common feeling already experienced by the users has been a high safety awareness. 


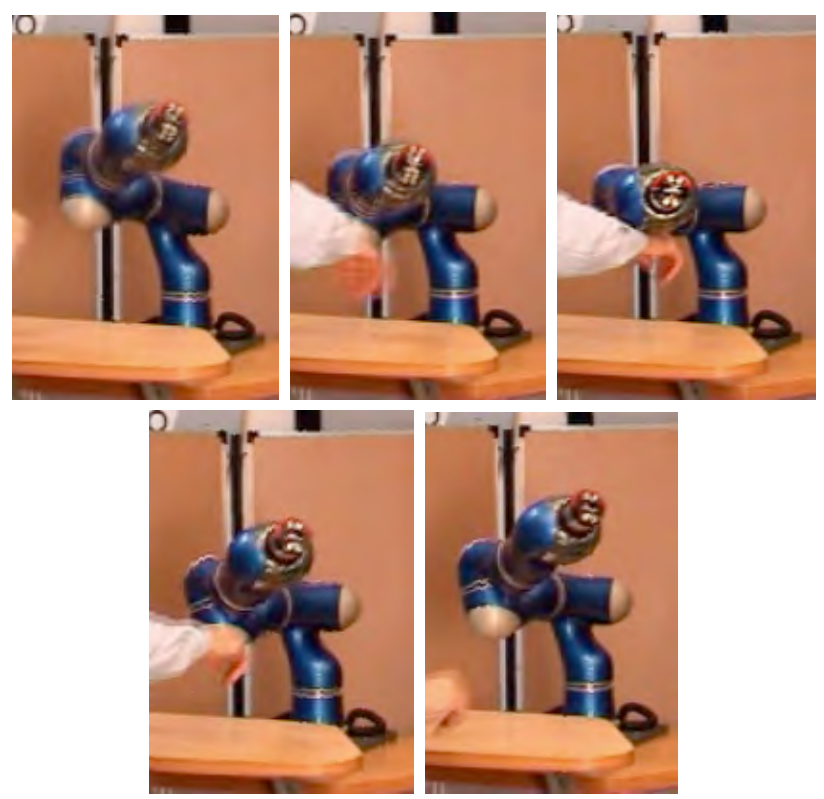

Fig. 7. Human-robot collision detection and reaction

\section{CONCLUSiOnS}

We have presented a complete approach, from detection to reaction, for handling human-robot collisions without the need of external sensing. Collision detection and identification signals can be efficiently generated resorting to energy arguments or based on the robot generalized momentum and by using only proprioceptive measurements. After collision has been detected, a reactive control strategy enables to reduce the effective inertia seen by the Cartesian contact forces. The robot retracts itself safely and rapidly away from the collision area, using the local directional information collected during the impact. The developed methodology covers both the case of rigid manipulators and of robots with elastic joints.

On-going work is concerned with acceleration-driven collision detection and the reduction of control communication delays in our robotic set-up, as well as with a more accurate evaluation of several severity indices of the impacts and of the beneficial inclusion of compliant coverages. Furthermore, robot redundancy will be exploited in order to devise reaction strategies that try to complete a given Cartesian motion task, despite of the detected collision.

\section{ACKNOWLEDGMENTS}

This work was performed while the first author was visiting DLR, with the support of a Research Award for foreign researchers granted by the Helmholtz-Humboldt Association.

\section{REFERENCES}

[1] J. Heinzmann and A. Zelinsky, "Quantitative safety guarantees for physical human-robot interaction," Int. J. of Robotics Research, vol. 22, no. 7/8, pp. 479-504, 2003.

[2] K. Ikuta, H. Ishii, and M. Nokata, "Safety evaluation method of design and control for human-care robots," Int. J. of Robotics Research, vol. 22, no. 7/8, pp. 281-297, 2003
[3] V. Lumelsky and E. Cheung, "Real-time collision avoidance in teleoperated whole-sensitive robot arm manipulator," IEEE Trans. on Systems, Man, and Cybernetics, vol. 23, no. 1, pp. 194-203, 1993.

[4] D. M. Ebert and D. D. Henrich, "Safe human-robot-cooperation: Imagebased collision detection for industrial robots," in Proc. IEEE/RSJ Int. Conf. on Intelligent Robots and Systems, pp. 1826-1831, 2002.

[5] G. Hirzinger, A. Albu-Schäffer, M. Hähnle, I. Schaefer, and N. Sporer, "On a new generation of torque controlled light-weight robots," in Proc. IEEE Int. Conf. on Robotics and Automation, pp. 3356-3363, 2001.

[6] Y. Yamada, Y. Hirasawa, S. Huang, Y. Uematsu, and K. Suita, "Humanrobot contact in the safeguarding space," IEEE/ASME Trans. on Mechatronics, vol. 2, no. 4, pp. 230-236, 1997.

[7] A. Bicchi and G. Tonietti, "Fast and soft arm tactics: Dealing with the safety-performance tradeoff in robot arms design and control," IEEE Robotics and Automation Mag., vol. 11, no. 2, pp. 22-33, 2004.

[8] M. Zinn, O. Khatib, B. Roth, and J. K. Salisbury, "A new actuation approach for human-friendly robot design," Int. J. of Robotics Research, vol. 23 , no. 4/5, pp. 379-398, 2005.

[9] A. De Luca, B. Siciliano, and L. Zollo, "PD control with on-line gravity compensation for robots with elastic joints: Theory and experiments," Automatica, vol. 41, no. 10, pp. 1809-1819, 2005.

[10] G. Tonietti, R. Schiavi, and A. Bicchi, "Design and control of a variable stiffness actuator for safe and fast physical human/robot interaction," in Proc. IEEE Int. Conf. on Robotics and Automation, pp. 528-533, 2005.

[11] S. Takakura, T. Murakami, and K. Ohnishi, "An approach to collision detection and recovery motion in industrial robot," in Proc. 15th Annual Conf. of IEEE Industrial Electronics Society (IECON89), (Boston, MA), pp. 421-426, 1989.

[12] K. Suita, Y. Yamada, N. Tsuchida, K. Imai, H. Ikeda, and N. Sugimoto, "A failure-to-safety 'kyozon' system with simple contact detection and stop capabilities for safe human-autonomous robot coexistence," in Proc. IEEE Int. Conf. on Robotics and Automation, pp. 3089-3096, 1995.

[13] K. Kosuge and T. Matsumoto, "Collision detection of manipulator based on adaptive control law," in Proc. IEEE/ASME Int. Conf. on Advanced Intelligent Mechatronics, pp. 117-122, 2001.

[14] S. Morinaga and K. Kosuge, "Collision detection system for manipulator based on adaptive impedance control law," in Proc. IEEE Int. Conf. on Robotics and Automation, pp. 1080-1085, 2003.

[15] A. De Luca and R. Mattone, "Sensorless robot collision detection and hybrid force/motion control," in Proc. IEEE Int. Conf. on Robotics and Automation, pp. 1011-1016, 2005.

[16] A. De Luca and R. Mattone, "Actuator fault detection and isolation using generalized momenta," in Proc. IEEE Int. Conf. on Robotics and Automation, pp. 634-639, 2003.

[17] H.-B. Kuntze, C. W. Frey, K. Giesen, and G. Milighetti, "Fault tolerant supervisory control of human interactive robots," in Proc. IFAC Work. on Advanced Control and Diagnosis (ACD03), (Duisburg, D), pp. 55-60, 2003.

[18] A. Albu-Schäffer, C. Ott, and G. Hirzinger, "A passivity based cartesian impedance controller for flexible joint robots - Part II: Full state feedback, impedance design and experiment," in Proc. IEEE Int. Conf. on Robotics and Automation, pp. 2666-2672, 2004.

[19] M. W. Spong, "Modeling and control of elastic joint robots," ASME J. of Dynamic Systems, Measurements, and Control, vol. 109, no. 4, pp. 310-319, 1987.

[20] A. De Luca and P. Lucibello, "A general algorithm for dynamic feedback linearization of robots with elastic joints," in Proc. IEEE Int. Conf. on Robotics and Automation, pp. 504-510, 1998.

[21] A. De Luca and R. Mattone, "An adapt-and-detect actuator FDI scheme for robot manipulators," in Proc. IEEE Int. Conf. on Robotics and Automation, pp. 4975-4980, 2004.

[22] A. De Luca, "Feedforward/feedback laws for the control of flexible robots," in Proc. IEEE Int. Conf. on Robotics and Automation, pp. 233 240, 2000.

[23] A. Albu-Schäffer and G. Hirzinger, "A globally stable state-feedback controller for flexible joint robots," Advanced Robotics, vol. 15, no. 8, pp. 799-814, 2001.

[24] C. Ott, A. Albu-Schäffer, A. Kugi, S. Stramigioli, and G. Hirzinger, "A passivity based cartesian impedance controller for flexible joint robots Part I: Torque feedback and gravity compensation," in Proc. IEEE Int. Conf. on Robotics and Automation, pp. 2659-2665, 2004.

[25] S. Haddadin, "Bewertungskriterien und Regelungsstrukturen für eine sichere Mensch-Roboter Interaktion," diplomarbeit, DLR, Oberpfaffenhofen, D, January 2006. 